\title{
COMPARISON OF EFFECTIVENESS OF BAL [BRONCHOALVEOLAR LAVAGE] WITH CT GUIDED FNAC IN THE DIAGNOSIS OF LUNG CANCER
}

\author{
Pradeep Kumar L1, Rudramurthy K. G², Srinivasa Murthy33, Avanthi E4
}

\section{HOW TO CITE THIS ARTICLE:}

Pradeep Kumar L, Rudramurthy K. G, Srinivasa Murthy, Avanthi E. "Comparison of Effectiveness of BAL [Bronchoalveolar Lavage] with CT Guided FNAC in the Diagnosis of Lung Cancer". Journal of Evolution of Medical and Dental Sciences 2014; Vol. 3, Issue 11, March 17; Page: 2752-2756,

DOI: $10.14260 /$ jemds/2014/2204

\begin{abstract}
The use of cytological methods in the diagnosis of lung cancer has been acclaimed as one of its most successful application. BAL has begun to play a more important role in the diagnosis of lung cancer. CT guided FNAC is particularly valuable in the diagnosis of lesions located in the periphery of the lung and in the mediastinum. AIMS: To study and compare the efficacy of these two cytological techniques i.e. BAL and CT guided FNAC in diagnosing lung cancer by correlating them with histopathological diagnosis by bronchoscopic biopsy. MATERIALS AND METHODS: A two year study was carried out. CT guided FNAC, BAL and bronchoscopic biopsies were done in 97 cases. Comparison of effectiveness of BAL and CT guided FNAC in diagnosing lung cancer is done with ' $\mathrm{z}$ ' test. RESULTS: Total 97 cases were included in the study, 33 cases were positive for malignancy. Sensitivity of BAL and CT FNAC were $69.6 \%$ and $100 \%$ respectively. On applying Z test for statistical analysis $\mathrm{p}$ value of $<0.0001$ is obtained. Hence we conclude that there is a significant difference in proportions between BAL and CT FNAC in diagnosing lung cancer. So CT FNAC is the most sensitive diagnostic modality for lung cancer. CONCLUSION: CT FNAC is much superior technique in the diagnosis and morphological classification of lung cancer in comparison to BAL.
\end{abstract}

KEYWORDS: Carcinoma lung; BAL; bronchoscopy; CT guided FNAC.

INTRODUCTION: The use of cytological methods in the diagnosis of lung cancer has been acclaimed as one of its most successful application. Flexible fiberoptic bronchoscope revolutionized the cytological diagnostic techniques in lung cancer in modalities like bronchoalveolar lavage (BAL) and bronchoscopic brush cytology. ${ }^{1}$ BAL has begun to play a more important role in the diagnosis of lung cancer. BAL specimen may also be used for molecular analysis in search of diagnostic and prognostic marker. ${ }^{2}$

CT guided FNAC is particularly valuable in the diagnosis of lesions located in the periphery of the lung and in the mediastinum. ${ }^{2}$

Our aim was to study and compare the efficacy of these two cytological techniques i.e. BAL and CT guided FNAC in diagnosing lung cancer by correlating them with his to pathological diagnosis by bronchoscopic biopsy.

MATERIAL AND METHODS: The study was carried out in the department of pathology at ESICMCPGIMSR, Rajajinagar, Bangalore. Out of all clinically and radiologically suspected cases of lung cancer received from June 2010 to June 2012(two years) i.e. 107 cases, we selected 97 cases where Bronchoalveolar lavage, CT guided FNAC and Bronchoscopic biopsy were available. The cases with inadequate samples in any of the above mentioned modalities were excluded. 
The samples were obtained by flexible fibre optic bronchoscopy done by pulmonologist. Bronchoalveolar lavage sample is sent in sterile vials as $20 \mathrm{ml}$ aliquots of normal saline, samples centrifuged and smears are prepared. Bronchial biopsy is sent in $10 \%$ formalin processed in routine tissue processor.

CT guided FNAC was done with 20G lumbar puncture needle; aspirate is directly smeared over the slide and fixed in alcohol. Slides are stained with H\&E, PAP, MGG and examined thoroughly for malignant cells.

The results of the smears were reported as:

(a) Normal/Negative for malignancy, (b) Inflammatory lesions, (c) Squamous metaplasia, (d) Positive for malignancy. ${ }^{3} \mathrm{Z}$ - test is applied for statistical analysis.

RESULTS: We included 97 patients with clinically suspicious of lung cancer. Out of 97 cases 33 cases were subsequently confirmed as lung cancer by bronchoscopic biopsy which was considered as gold standard in our study. Out of 97 cases 79 were male and 18 were female with their age ranging from 50-79yrs. The male: female ratio was 4.3:1. A history of smoking more than 15 cigarettes per day for more than five years was given by 32 patients with lung cancer, thus we have a striking majority of 96\% smokers in lung cancer group.

Radiological and bronchoscopic studies revealed a central tumor in 11 cases and peripheral in 22. Most of the central tumors were of squamous cell carcinoma showing malignant squamous cells in BAL (Fig No.1) as well as in CT FNAC. (Fig No.2)

One case was showing features of small cell carcinoma in BAL as well as in CT FNAC which was later confirmed by immunohistochemistry i.e. chromogranin and synaptophysin on biopsy. BAL was positive in 23 cases (69.3\%) and CT FNAC was positive in all 33 cases with $100 \%$ sensitivity. There were no false positive cases.

Distribution of different types of lung cancers and number of positive cases with each diagnostic modality is described in table no.1 and 2 .

Thus in these 33 cases the following observations were made.

Sensitivity of BAL cytology $-69.6 \%$

Sensitivity of CT FNAC $-100 \%$

On applying Z test for statistical analysis $\mathrm{p}$ value of $<0.0001$ is obtained. Hence we conclude that there is a significant difference in proportions between BAL and CT FNAC in diagnosing lung cancer. So CT FNAC is the most sensitive diagnostic modality for lung cancer.

DISCUSSION: Advent of flexible fiberoptic bronchoscope has led to new turn as samples like lavage, washings, brush smear, transbronchial needle aspiration and biopsy can be collected from the respiratory tract. ${ }^{4}$

BAL is a valuable diagnostic and research tool in pulmonology. ${ }^{5}$ In a comparative study of BAL and open lung biopsy Yamamoto found that the results of these two to have a parallel relation except in few cases. ${ }^{6}$ Techniques of conventional respiratory cytology and FNAC are complimentary in the diagnosis of lung cancer. ${ }^{7}$

In our study in comparison to BAL, CT FNAC has a higher number of true positive and true negative cases with sensitivity of $100 \%$, showing its superiority over BAL in diagnosing lung cancer.(table-2) 
Cytological sampling by BAL relies mainly on cells exfoliated from malignant lesion in bronchial epithelium. ${ }^{4}$ CT FNAC has the advantage that the cells are aspirated from the lesion proper. Then the chance of getting adequate cytological sample by CT FNAC is increased in comparison to BAL sampling. ${ }^{4}$

In our study sensitivity of BAL samples is $69.6 \%$ which is comparable to other studies such as that of Jay S J (1980) with 63\%, 8 Sing A (1997) with 50\% ${ }^{9}$ and Gaur D S (2012) with 39.4\% sensitivity. 1

In our study sensitivity of CT FNAC is $100 \%$ which is comparable to that of Guliamos A $D(2000)$ study with $98 \%$ sensitivity, ${ }^{10} 0^{\prime}$ reilly $P$ E(1994) study with $68 \%$ sensitivity ${ }^{11}$ and Johnston(1988) with sensitivity of $72.6 \% .^{4}$

Luckily our study has no false positive cases.

If cytology is positive for malignancy or suspicious cells repeat biopsy, clinical correlation with radiological and bronchoscopic findings is necessary. ${ }^{12}$

In the present study majority of the cases are of squamous cell carcinoma followed by adenocarcinoma and other types. Squamous cell carcinoma which is more frequently located in proximal bronchus showed positivity in 13 cases through BAL cytology.

Wongsurakiat et al found that the diagnostic yield of BAL is influenced by the size and segmental location of the lesion. ${ }^{13}$

With good sensitivity CT FNAC promises to be a convenient cytological technique that can be confidently utilized for the screening of doubtful cases and early diagnosis of lung cancer as it saves the time needed for the processing of the biopsy specimens. ${ }^{14}$

CONCLUSION: CT FNAC is much superior technique in the diagnosis and morphological classification of lung cancer in comparison to BAL.

\section{REFERENCES:}

1. Gaur DS, Thapliyal NC, Kishore S, Pathak VP. Efficacy of bronchoalveolar lavage and bronchial brush cytology in diagnosing lung cancers. J Cytol 2007; 24:73-7.

2. Myron R Melamed, Leopard G Koss. Tumors of Lung. In: Myron R Melamed, Leopard G Koss, editor. Koss' diagnostic cytology and its histopathological bases, $5^{\text {th }}$ ed. Philadelphia: Lippincott Williams \& Wilkins; 2009. p. 645-706.

3. Ammanagi AS, Dombale VD, Miskin AT, Dandagi GL, Sangolli SS. Sputum cytology in suspected cases of carcinoma of lung (Sputum cytology a poor man's bronchoscopy!).Lung India 2012; 29:19-23.

4. Johnston WW, Elson CE. Respiratory tract. In: Bibbo M, editor. Comprehensive cytopathology, $2^{\text {nd }}$ ed. Philidelphia: W. B. Saunders Company; 1997. p. 325-401.

5. Kopinski P, Chlap Z, Owsinski J, Soja J, Stankiewicz Z, Biernat-Silczuk M, et al. Principles of optimal preparation of material from bronchoalveolar lavage (BAL)for cytoimmunologic examinations in interstitial lung diseases. PrzeglLek 2000; 57: 489-92.

6. Yamamoto S. Diagnostic value of bronchoalveolar lavage (BAL) - the comparative study with open lung biopsy and BAL. Rinsho Byori 1994; 42: 265-70.

7. Johnston WW. Fine needle aspiration biopsy versus sputum and bronchial material in the diagnosis of lung cancer-A comparative study of 168 patients. Acta Cytol 1988; 32: 641-6. 
8. Jay SJ, Wehr K, Nicholson DP, Smith AL. Diagnostic sensitivity and specificity of pulmonary cytology: comparison of techniques used in conjunction with flexible fibre optic bronchoscopy. Acta Cytol 1980; 24: 304-12.

9. Sing A, Freudenberg N, Kortsik C, Wertzel H, Klosa B, Hasse J. Comparison of the sensitivity of sputum and brush cytology in the diagnosis of lung carcinomas. Acta Cytol 1997; 41: 399-408.

10. Gouliamos AD, Giannopoulos DH, Panagi GM, Fletoridis NK, Deligeorgi-Politi HA, Vlahos LJ. Computed tomography-guided fine needle aspiration of peripheral lung opacities. An initial diagnostic procedure? Acta Cytol 2000; 44: 344 -8.

11. O'Reilly PE, Brueckner J, Silverman JF. Value of ancillary studies in fine needle aspiration cytology of the lung. Acta Cytol 1994; 38: 144-50.

12. Ahmed A, Ahmed S. Comparison of bronchoalveolar lavage cytology and transbronchial biopsy in the diagnosis of carcinoma of lung. J Ayub Med College Abbottabad 2004; 16: 29-33.

13. Wongsurakiat P, Wongbunnate S, Dejsomritrutai W, Charoenratanakul S, Tscheikuna J, Youngchaiyud P, et al. Diagnostic value of bronchoalveolar lavage and post bronchoscopic sputum cytology in peripheral lung cancer. Respirology 1998; 3: 131-7.

\begin{tabular}{|l|c|c|}
\hline & Number of cases & $\mathbf{\%}$ \\
\hline Squamous cell carcinoma. & 20 & 60.6 \\
\hline Adenocarcinoma. & 07 & 21.2 \\
\hline Small cell carcinoma. & 02 & 06.06 \\
\hline Large cell carcinoma. & 01 & 03 \\
\hline Carcinoid. & 01 & 03 \\
\hline Lymphoma & 01 & 03 \\
\hline Plasmacytoma & 01 & 03 \\
\hline \multicolumn{1}{|c|}{ Total } & $\mathbf{3 3}$ & \\
\hline
\end{tabular}

Table 1: Distribution of different types of lung cancer

\begin{tabular}{|c|c|c|c|}
\hline & BAL* & CT FNAC $\dagger$ & Biopsy \\
\hline Squamous cell carcinoma. & 13 & 20 & 20 \\
\hline Adenocarcinoma. & 07 & 07 & 07 \\
\hline Small cell carcinoma. & 01 & 02 & 02 \\
\hline Large cell carcinoma. & 00 & 01 & 01 \\
\hline Carcinoid. & 01 & 01 & 01 \\
\hline Lymphoma & 00 & 01 & 01 \\
\hline Plasmacytoma & 01 & 01 & 01 \\
\hline Total & $23(69.6 \%)$ & $33(100 \%)$ & 33 \\
\hline
\end{tabular}

*Bronchoalveolar lavage, † CT guided fine needle aspiration cytology. 


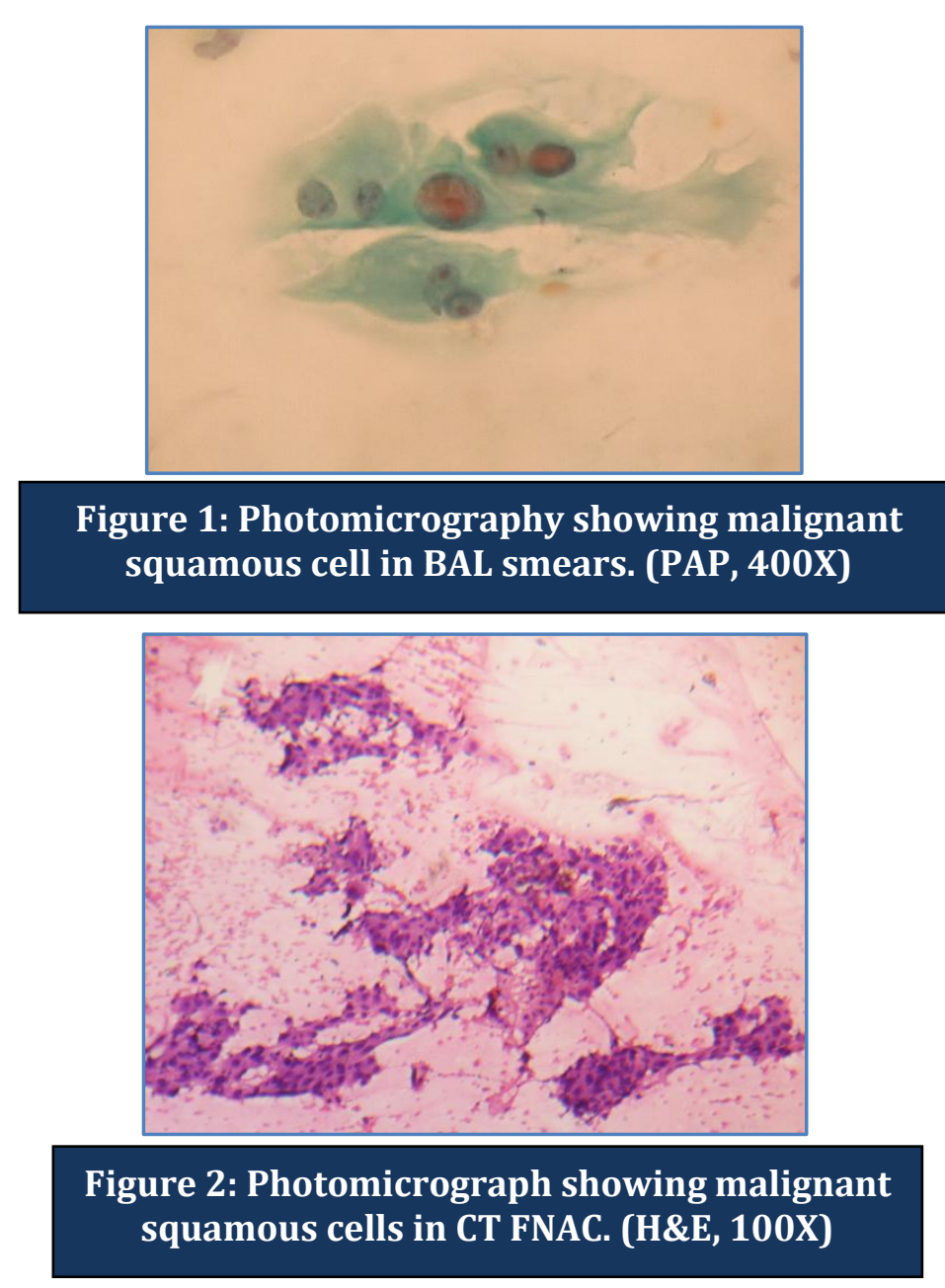

\section{AUTHORS:}

1. Pradeep Kumar L.

2. Rudramurthy K. G.

3. Srinivasa Murthy

4. Avanthi E.

\section{PARTICULARS OF CONTRIBUTORS:}

1. Assistant Professor, Department of Pathology, Karuna Medical College, Palakkad, Kerala.

2. Assistant Professor, Department of Microbiology, Karuna Medical College, Palakkad, Kerala.

3. Professor and HOD, Department of Pathology, ESICMCPGIMSR, Bangalore.

4. Post Graduate Tutor, Department of Pharmacology, JJMMC, Davangere. 